\title{
Integration of AYUSH with Dentistry
}

Various forms of age-old medicine are still in practice in several clusters of regions and communities in India especially in the treatment of common chronic ailments. Among these, Ayurveda is continued to be one of most ancient medicine which provides healthcare with a remarkable balance between patient safety and efficacy. Other medicine systems like Yoga, Unani, Siddha, and Homeopathy are also practiced to cater to good health to society. These medicine systems along with naturopathy are providing unremitting health care to all age groups of patients in the prevention and treatment of various diseases and disorders. These traditional medicines apply holistic approaches which include diverse health practices, approaches, knowledge and beliefs incorporating plant, animal, and/or mineralbased medicines, spiritual therapies, manual techniques, and exercises applied singularly or in combination to maintain well being, not only in diagnosis or treatment but also in the prevention of illness. In 1995, Govt of India established a department which was later on in 2003 renamed as AYUSH (an acronym for Ayurveda, Yoga and Naturopathy, Unani, Siddha and Homeopathy) with an aim to expand the dimension of these medicine systems. In recent years, the Govt of India has taken some milestone steps to promote it by integrating AYUSH with local health traditions in the National Rural Health Mission (NRHM). National Health policy (2017) strongly recommended the potential role of AYUSH in the Integrated health care system.

In Oral health, the use of herbal medicine has been applied in clinical trials as antiseptic, analgesics and antioxidants. In addition, few natural phytochemicals have been identified as an alternative to antibiotics in the healing of oral infections and thereby improving immunity. In the published literature, there is ample evidence of randomized clinical trials on the use of herbal and homeopathic medicine in various chronic and precancerous disorders of the oral cavity. There are few examples in this context i.e. a) use of herbal extract against the cariogenic bacteria, in healing of periodontal tissues, against fungal infections and in treatment of oral potentially malignant disorders b) use of homeopathic medicine in inflammatory disease, in reduction dental anxiety, dental pain and healing of recurrent and chronic ulcers and many more are still under trials.

When the current world is concerned about the adverse effects of conventional medicines including antibiotic resistance especially in the management of chronic diseases, these complementary or allied medicine systems could be a ray o hope because of their therapeutic benefits including immense safety and efficacy. In a country like India which is thought to home to many ancient and traditional medicine should step forward to support and integrate traditional medicine with conventional one to ensure the patient's complete wellness. On one hand, scientific validation and evidence-based clinical trials on these allied medicines should be carried out in dental fields, on other hand awareness among the general population about the benefits of the same should be highlighted to gain confidence for use in prevention and treatment of dental disorders. It is also essential for health professionals to have a sound knowledge of complementary and alternative modalities for the treatment of these chronic ailments. These efforts by various stake holders could help us to make dentistry much safer, affordable and more accessible all strata of population irrespective of their socioeconomic status. 